\title{
THIRD GENERAL CATALOGUE OF STARS OBSERVED WITH THE PHOTOELECTRIC ASTROLABES
}

\author{
Lu Lizhi
}

(Beijing Astronomical Observatory in China)

\begin{abstract}
On the basis of the data observed with the Photoelectric Astrolabes in China observed during the period from 1982 to 1990 , the general catalogue is compiled. With magnitudes ranging from $0^{m} .1$ to $7^{m} .3$, the general catalogue consists of 2577 stars spreading from $\delta=$ $-3^{\circ}$ to $\delta=69^{\circ}$, in which 955 are FK5 stars and 1622 are GC stars. The mean precisions of $2577 \Delta \alpha$ 's and $1892 \Delta \delta$ 's are $\pm 4.0 \mathrm{~ms}$ and $\pm 0 " .065$, respectively. The mean epoch of the catalogue is 1987.4. Finally systematic corrections of (CAT-FK5) are given.
\end{abstract}

\section{Introduction}

The equal altitude method brings good results not only for the determination of the Earth's rotation parameters, but also in the improvement of catalogues. The potentiality of this method in the field of position astronomy has been shown by several general catalogue [1.2] of stars observed with the different marks of chinese photoelectric astrolabes since 1973. The general catalogue reported here is compiled on the basis of the preliminary catalogue of the photoelectric astrolabe mark II of Beijing, Yunnan and Shanghai Astronomical Observatories and mark I photoelectric astrolabe of Shaanxi Astronomical Observatory since 1982.

The chinese photoelectric astrolabes mark II have been modified to automatic ones and photo-counters are used for recording the time of almucanter transit of a star with the limiting magnitude of at least $9^{m} .5$. The photoelectric astrolabe mark III are adjusting and will be installed at Beijing, Yunnan Astronomical Observatories respectively. These instruments will be used for observations of faint stars and some minor planets.

\section{Reduction of the data}

Adopting the positions at J2000.0 of the Fifth Fundamental Catalogue (FK5) and new astronomical constants (IAU, 1976), the data observed with the Photoelectric Astrolabes are reduced to the FK5 system.

All preliminary catalogue are compiled using same method. The equation defined the position corrections are

$$
\Delta \alpha=\frac{V_{e}-V_{w}}{30 \cos \varphi_{0}|\sin A|}+\Delta A
$$




$$
\Delta \delta=-\frac{V_{e}+V_{w}-2 K}{2 \cos q}+D \cos \delta
$$

where

$V_{e}, V_{w}$ - the residuals reduced to the mean instrumental system at both eastern and western passages;

$\varphi_{0}$ - the adopted value of latitude at the site of the instrument;

$A$ - the azimuth of a star observed, measured eastwards from north ;

$q$ - the parallactic angle of a star as it transits the almucantar of the astrolabe.

$\Delta \mathrm{A}$ and $\mathrm{D} \cos \delta$ are constans to be determined. $2 \mathrm{~K}$ can be calculated using the stars of $|\cos q|<0.2$.

After correcting for the magnitude equations, spectral type equations, difference in the constant of right ascensions, the value of $2 \mathrm{~K}$ 's and the systematic error of delclinations of the form $\mathrm{D} \cos \delta$, the analytic method developed by R.Bien et al ${ }^{[3]}$ have been used to obtain the systematic differences respectively of $\alpha, \delta$ and magnitude $\mathrm{M}$ in the sence of (CAT-FK5) for each preliminary catalogues. The systematic difference may be given by

$$
f_{i}(\delta, M, \alpha)=R_{p n m l} L_{n}(X<\delta>) J_{p}(Y<M>) F_{m l}(\alpha)
$$

where

$R_{\text {pnml }}$ - normalizing factor;

$L_{n}(X<\delta>)$ - Lgendre polynomial;

$J_{p}(Y<M>)$ - Jacobi polynomial;

$F_{m l}(\alpha)$ - Fourier term.

Meanwhile, a computerized numerical method has been used as a comparison. It is considered that these results ara agree with each other quite well.

The composite system of the general catalogue is formed by weighting each FK5 star of different preliminary catalogue according to their systematic differences

$$
f_{i}(X<\delta>, Y<M>, \alpha)
$$

And all GC stars have been obtained with the same process.

\section{The Results}

The general catalogue consits of 2577 stars in which there are 955 FK5 stars and 1622 GC stars. The magnitudes are from $0^{m} .1$ to $7^{m} .3$. The declinations are from $\delta=-3^{\circ}$ to $\delta=69^{\circ}$. The mean precisions of $2577 \Delta \alpha$ 's and $1892 \Delta \delta^{\prime}$ 's are $\pm 4.0 \mathrm{~ms}$ and $\pm 0^{\prime \prime} .065$, respectively. In the catalogue, among these stars appearing in more than one preliminary 
catalogue, there are 885 common in $\Delta \alpha$ and 369 in $\Delta \delta$. The mean accordances for the common stars in $\Delta \alpha$ and $\Delta \delta$ are $\pm 4.5 \mathrm{~ms}$ and $\pm 0^{\prime \prime} .061$, respectively. The mean epoch of the catalogue with 188 thousants observations is 1987.4 .

By the method [1] and [3] and with the $\Delta \alpha$ and $\Delta \delta$ of FK5 stars, the systematic corrections of the catalogue of stars (CAT-FK5) are analyzed. The systematic corrections on the right ascension, declination, and magnitude $\Delta \alpha_{\alpha}, \Delta \delta_{\alpha}, \Delta \alpha_{\delta}, \Delta \delta_{\delta}, \Delta \alpha_{m}$, and $\Delta \delta_{m}$, are given in Tables 1, 2, and 3, respectively.

Table 1. The Systematic Errors (CAT-FK5) $\Delta \alpha_{\alpha}$ and $\Delta \delta_{\alpha}$

\begin{tabular}{||c|rrrrrrrrrrrr||}
\hline$\alpha^{h}$ & 0 & 1 & 2 & 3 & 4 & 5 & 6 & 7 & 8 & 9 & 10 & 11 \\
\hline$\Delta \alpha_{\alpha}\left(0^{3} .0001\right)$ & 0 & 7 & 12 & 16 & 17 & 16 & 13 & 9 & 5 & 2 & 0 & 0 \\
$\Delta \delta_{\alpha}\left(0^{\prime \prime} .001\right)$ & 17 & 28 & 15 & -5 & -12 & 1 & 7 & 16 & -5 & -29 & -32 & -11 \\
\hline \hline$\alpha^{h}$ & 12 & 13 & 14 & 15 & 16 & 17 & 18 & 19 & 20 & 21 & 22 & 23 \\
\hline$\Delta \alpha_{\alpha}\left(0^{5} .0001\right)$ & 0 & 0 & 0 & -2 & -5 & -9 & -13 & -16 & -17 & -16 & -12 & -7 \\
$\Delta \delta_{\alpha}\left(0^{\prime \prime} .001\right)$ & 17 & 28 & 15 & -5 & -12 & 1 & 7 & 16 & -5 & -29 & -32 & -11 \\
\hline
\end{tabular}

Table 2. The Systematic Errors (CAT-FK5) $\Delta \alpha_{\delta}$ and $\Delta \delta_{\delta}$

\begin{tabular}{||c|rrrrrrr||}
\hline$\delta^{\circ}$ & 0.0 & 5.0 & 10.0 & 15.0 & 20.0 & 25.0 & 30.0 \\
\hline$\Delta \alpha_{\delta}\left(0^{s} .0001\right)$ & -3 & 6 & -29 & -14 & 22 & 29 & 12 \\
$\Delta \delta_{\delta}\left(0^{\prime \prime} .001\right)$ & 47 & 45 & 19 & -3 & -11 & -9 & -4 \\
\hline \hline$\delta^{\circ}$ & 35.0 & 40.0 & 45.0 & 50.0 & 55.0 & 60.0 & 65.0 \\
\hline$\Delta \alpha_{\delta}\left(0^{3} .0001\right)$ & 3 & 8 & 11 & 3 & -7 & -16 & -50 \\
$\Delta \delta_{\delta}\left(0^{\prime \prime} .001\right)$ & -3 & -10 & -29 & -33 & -38 & -31 & -9 \\
\hline
\end{tabular}

Table 3. The Systematic Errors (CAT-FK5) $\Delta \alpha_{m}$ and $\Delta \delta_{m}$

\begin{tabular}{||c|rrrrrrr||}
\hline $\bar{M}$ & 0.33 & 2.14 & 3.11 & 4.02 & 5.04 & 5.94 & 6.76 \\
\hline$\Delta \alpha_{m}\left(0^{3} .0001\right)$ & -9 & 1 & -3 & 0 & 0 & -2 & -8 \\
\hline \hline $\bar{M}$ & 0.06 & 2.16 & 3.08 & 4.01 & 5.04 & 5.91 & 6.73 \\
\hline$\Delta \delta_{m}\left(0^{\prime \prime} .001\right)$ & 6 & -13 & 3 & 3 & 0 & 1 & 1 \\
\hline
\end{tabular}

\section{References}

[1] Working Group of GCPA, Acta Astronomica Sinica, 24 (1983), 267. (In chinese)

[2] Zhu Yuanxen, Lu Lizhi et al. Publications of the Beijing Astronomical Observatory, No.1 (1979), 56.(In chinese)

[3] Bien, R.,Veröff. Astron. Rechen-Institut, Heidelberg, No.29 (1978).

[4] Lu Lizhi. Publications of the Beijing Astronomical Observatory, No.14 (1989) 50. (In english) 